\title{
ITRAQ-based comparative proteomic analysis in mature leaves of Ethiopian mustard (Brassica carinata) provides insights into the whole-proteomic profiles and the role of anthocyanin biosynthesis in leaf color diversity
}

Tianya Wang

Guizhou University

Shaomin Guo

Guizhou Academy of Agricultural Science

Kunjiang Yu

Guihzou University

Jun Zou

Huazhong Agriculture University

Yingfen Jiang

Anhui Academy of Agricultural Sciences

Wei Wan

Guizhou University

Botao Ye

Guizhou University

Renqin Yang

Guizhou University

Aimal Nawaz Khattak

Guizhou University

Jinling Meng

Huazhong Agriculture University

Entang Tian ( $\nabla$ erictian121@163.com )

Guizhou University https://orcid.org/0000-0002-9039-3666

Research article

Keywords: Brassica carinata, anthocyanin, iTRAQ, proteomic analysis, purple leaves

Posted Date: September 12th, 2019 
DOI: https://doi.org/10.21203/rs.2.14299/v1

License: (c) (1) This work is licensed under a Creative Commons Attribution 4.0 International License. Read Full License 


\section{Abstract}

Background Anthocyanins are the main pigments in leaves, flowers and fruits, performing diverse biological functions in plants and exhibiting benefits for human health. The purple-leaved accession of $B$. carinata was used to study the biosynthetic mechanism of anthocyanins in this study. To elucidate the mechanisms of anthocyanin accumulation in the purple-leaved line, we employed a proteomic approach based on an isobaric tags for relative and absolute quantification (iTRAQ)-based technique to investigate the protein expression profile of the purple-leaved line of B. carinata in comparison with that of the greenleaved line.

Results In total, 4,631 proteins were identified, of which 285 exhibited significant changes in abundance between BC-G01 and BC-P01. Of the 285 DEPs, 175 are upregulated and 110 are downregulated in the leaves of the purple-leaved line compared with the leaves of the green-leaved line. Bioinformatics analysis indicated that anthocyanin biosynthesis was the most significantly elevated metabolic process, containing three DEPs corresponding to two genes UGT75C1 and UGT79B1. UGT75C1 and UGT79B1, encoding anthocyanin 3-0-glucoside: 2"-0-xylosyltransferase and anthocyanin 5-0-glucosyltransferase, respectively, play an important role in the biosynthesis of anthocyanins.

Conclusions The exact functions of these proteins remain to be examined. Nevertheless, our study paves the way for understanding the genetic regulatory mechanisms of anthocyanin biosynthesis and accumulation in B. carinata .

\section{Background}

Brassica carinata $(B B C C, 2 n=34)$ is an important vegetable and oilseed crop in northeast Africa and is thought to have originated in the Ethiopian plateau [1-3]. Together with another two amphidiploid Brassica species, namely, $B$. napus (AACC, $2 \mathrm{n}=38$ ) and $B$. juncea (AABB, $2 \mathrm{n}=36$ ), in the U-triangle, $B$. carinata is an amphidiploid of recent origin $[2,4,5]$. Cytogenetic and molecular evidence suggests that $B$. carinata has evolved as a natural cross between $B$. oleracea $(C C, 2 n=18)$ and $B$. nigra $(B B, 2 n=16)$, followed by subsequent chromosome doubling [5-8]. The lengthy processes of speciation and domestication have resulted in diverse eco-types with the whole range of morphological and agronomic differences [8-10]. Given the many desirable characteristics of $B$. carinata, such as resistance to pests, tolerance to diseases and drought, and applicability as a bio-industrial oil crop [11, 12], we made great efforts to study and utilize the species, such as by transferring the yellow-seeded trait to $B$. napus from $B$. carinata [13], evaluating the genetic diversity of 110 accessions of $B$. carinata with emphasis on the interspecific crossability with $B$. rapa [10], synthesizing the Brassica trigenomic allohexaploid via $B$. carinatax $B$. rapa [14], generating the new-type $B$. napus by incorporating components of the $C$ genome of $B$. carinata into traditional $B$. napus $[15,16]$, assessing the genetic introgression from $B$. carinata into new-type $B$. napus breeding lines [17], construction of one double-haploid mapping population of $B$. carinata and QTL mapping of petal and anther tip color, seed coat color, flower development and quality 
traits $[2,18,19]$. As continued research, we attempted to exploit the proteomic profiles and identify the key genes associated with anthocyanin biosynthesis in purple-leaved $B$. carinata in this study.

Anthocyanins are the main pigments in leaves, flowers and fruits and are responsible for the red-to-blue colors of plant organs $[20,21]$. Approximately 17 anthocyanidins are found in nature, but only 6 are widely distributed: cyanidin, delphinidin, petunidin, peonidin, pelargonidin, and malvidin [21, 22]. The individual anthocyanidin content varies among species with respect to the following features: (i) the number and positions of hydroxyl $(\mathrm{OH})$ groups, (ii) the degree of methylation of these $\mathrm{OH}$ groups, (iii) the nature, number, and location of sugars attached to the molecule, and (iv) the nature and number of aliphatic or aromatic acids attached to the sugar [21,23]. In addition, the colorful appearance of different organs depends on the $\mathrm{pH}$ value of the anthocyanin solution, because of the ionic nature of the molecular structure of anthocyanins $[24,25]$. In the present study, the leaves of $B$. carinata appear purple; anthocyanins have a purple hue in neutral $\mathrm{pH}$, but the color changes to blue with increasing $\mathrm{pH}$ [25]. Natural mutants with purple organs have been found and reported in many species, such as in asparagus [26], crackers and bars of wheat [27], rice [28], eggplant [29], corn [30], carrot, potatoes [31], basil [32], tea [33], and pepper [34] and in the Brassica U-triangle species B. oleracea [35], B. napus [36], B. rapa [37], and $B$. juncea [38], in addition to $B$. nigra and $B$. carinata. The purple color resulting from the presence of anthocyanins in different species can be used as a natural food colorant, and these compounds have health-associated nutraceutical properties.

ITRAQ is a novel, MS-based approach for the relative quantification of proteins, relying on the derivatization of primary amino groups in intact proteins using an isobaric tag for relative and absolute quantitation [39]. This method has been widely used in the identification of key proteins related to target traits in recent years. In the present study, the high-throughput iTRAQ methodology was used to investigate protein and qualitative changes between the two double-haploid lines of $B$. carinata with purple and green leaves. To the best of our knowledge, this is the first report of proteomic research related to purple leaves in $B$. carinata. This study provides novel insights into the proteomic profile of $B$. carinata and for identification of the key protein involved in anthocyanin biosynthesis in this species.

\section{Methods}

\section{Plant materials}

Two B. carinata double-haploid (DH) lines, namely, BC-P01 and BC-G02, were used in this study. BC-P01 and BC-G02, originally named CGN03981 and CGN03976, respectively, were obtained from the Centre for Genetic Resources, Wageningen, the Netherlands (CGN, http://www.cgn.wur.nl/NL/) [10]. In October 2017, BC-P01 and BC-G02 were planted at the farm of Guizhou University, Guiyang, China. In December 2018, three randomly selected plants for each of BC-P01 and BC-G02 were chosen for picking of leaf samples. The fifth mature leaf from the ground for each plant was sampled and transferred into $a-80^{\circ} \mathrm{C}$ freezer for storage. 


\section{Determination of total anthocyanin content}

The total anthocyanin content was estimated by the simplified method and $\mathrm{pH}$ differential method, respectively. For the $\mathrm{pH}$ differential method, six freeze-dried leaf samples $(0.5 \mathrm{~g})$ were ground to a powder and then extracted twice with $25 \mathrm{ml}$ of methanol:water:acetic acid ( $\mathrm{V}: \mathrm{V}: \mathrm{V}=85: 15: 0.5)$ at $50{ }^{\circ} \mathrm{C}$ for $2 \mathrm{~h}$. The extract solution was collected and filtered through a $0.22 \mu \mathrm{m}$ filter to measure the absorbance according to Wang et al. [40] and Zhao et al. [38]. Determination of the absorbance of the extracts was performed with a SPECORD 200 PLUS UV spectrophotometer (Analytik Jena AG, Jena, CA). In our experiment, the optimum temperature was $50^{\circ} \mathrm{C}$, and the equilibration times for $\mathrm{pH} 1.0$ and pH 4.5 were $80 \mathrm{~min}$ and $100 \mathrm{~min}$, respectively. The total absorbance (A) was calculated as follows: $A=(A 510-A 700)$ $\mathrm{pH} 1.0-(\mathrm{A} 510-\mathrm{A} 700) \mathrm{pH}$ 4.5. Then, the total anthocyanin content $(T A C)$ was estimated as $\mathrm{C}\left(\mathrm{mg} \cdot \mathrm{g}^{-1}\right)=$ $(\mathrm{A} \times \mathrm{V} \times \mathrm{n} \times \mathrm{M}) /(\varepsilon \times \mathrm{m})[\mathrm{A}$ : the absorbance difference between $\mathrm{pH} 1.0$ and $\mathrm{pH} 4.5$; $\mathrm{V}$ : total volume of extract $(\mathrm{mL})$; $\mathrm{n}$ : dilution factor; $\mathrm{M}$ : relative molecular mass of cyanidin-3-glucoside (449.4); $\varepsilon$ : extinction coefficient of cyanidin-3-glucoside (26900); m: sample mass (g)] according to Wang et al. [40] and Cheng et al. [41]. For the simplified method, most steps were performed according to Wang et al. [42] and Yu et al. [43] with minor modifications. Determination of the absorbance of the extracts was performed on a SPECORD 200 PLUS UV spectrophotometer (Analytik Jena AG, Jena, CA). The anthocyanin content is expressed in $\mathrm{g} \cdot 10 \mathrm{ml} 0.1 \mathrm{~mol} / \mathrm{L}$ hydrochloric acid.

\section{Protein extraction for iTRAQ analysis}

Total proteins from the sixth samples were extracted according to the following procedure. Approximately $1.0 \mathrm{~g}$ of the ground-up leaf powder of each sample was combined with $4 \mathrm{ml}$ of $10 \% \mathrm{~m} / \mathrm{v}$ trichloroacetic acid (TCA) in acetone, and the samples were incubated at $-20^{\circ} \mathrm{C}$ for $2 \mathrm{~h}$. The samples were then centrifuged at $20,000 \times \mathrm{g}$ for $30 \mathrm{~min}$ at $4^{\circ} \mathrm{C}$. The supernatant was discarded without disturbing the pellets. To reduce acidity, the pellets were washed with acetone and incubated at $-20^{\circ} \mathrm{C}$ for $30 \mathrm{~min}$ and centrifuged at $20,000 \times \mathrm{g}$ for $30 \mathrm{~min}$ at $4^{\circ} \mathrm{C}$. The washing step with acetone was repeated several times until the pellets were white. The dried pellets were lysed with $1 \mathrm{ml}$ of protein extraction reagent [8 $\mathrm{M}$ urea, $30 \mathrm{mM}$ HEPES, 1 mM PMSF, $2 \mathrm{mM}$ EDTA and $10 \mathrm{mM} \mathrm{DTT}]$. The pellets were then dissolved by ultrasonication (pulse on $2 \mathrm{~s}$, pulse off $3 \mathrm{~s}$, power $180 \mathrm{w}$ ) for five minutes. After dissolution, the solution was centrifuged at $20,000 \times \mathrm{g}$ for $30 \mathrm{~min}$ at $4^{\circ} \mathrm{C}$ to remove insoluble impurities. Proteins were reduced with $10 \mathrm{mM} \mathrm{DTT}$ at $56^{\circ} \mathrm{C}$ for $1 \mathrm{~h}$ and alkylated immediately with $55 \mathrm{mM}$ iodoacetamide (IAM) in the dark at room temperature for $1 \mathrm{~h}$. The treated proteins were precipitated in acetone at $-20^{\circ} \mathrm{C}$ for $3 \mathrm{~h}$. After centrifugation at $20,000 \times \mathrm{g}$ for $30 \mathrm{~min}$ at $4^{\circ} \mathrm{C}$, the pellets were resuspended and ultrasonicated in prechilled $50 \%$ TEAB buffer with $0.1 \%$ SDS and dissolved by ultrasonication. The proteins were regained after centrifugation at $20000 \times \mathrm{g}$ for $30 \mathrm{~min}$, and the protein concentration was determined by the Bradford assay (Bio-Rad) using BSA as a standard.

\section{In-solution digestion and iTRAQ labeling}


For each sample, $100 \mu \mathrm{g}$ of protein in TEAB buffer was incubated with $3.3 \mu \mathrm{g}$ of trypsin $(1 \mu \mathrm{g} / \mu \mathrm{l})$ (Promega, Madison, WI, USA) at $37^{\circ} \mathrm{C}$ for $24 \mathrm{~h}$ in a sealed tube. The tryptic peptides were lyophilized and dissolved in 50\% TEAB buffer. Samples were labeled using the iTRAQ Reagents 8-plex Kit according to the manufacturer's instructions (AB Sciex Inc., MA, USA). A total of $100 \mu \mathrm{g}$ of protein in TEAB buffer was incubated with $3.3 \mu \mathrm{g}$ of trypsin $(1 \mu \mathrm{g} / \mu \mathrm{l})$ (Promega, Madison, WI, USA) at $37^{\circ} \mathrm{C}$ for $24 \mathrm{~h}$ in a sealed tube. The tryptic peptides were lyophilized and dissolved in 50\% TEAB buffer. Samples were labeled using the iTRAQ Reagents 8-plex Kit according to the manufacturer's instructions (AB Sciex Inc., MA, USA).

\section{HPLC (strong cation exchange)}

The labeled samples were then alkalified and fractionated using a strong cation exchange HPLC system (Agilent 1100, USA) connected to an SCX column (Luna $5 \mathrm{u}$ column, $4.6 \mathrm{~mm} \times 250 \mathrm{~mm}, 5 \mu \mathrm{m}, 100 \AA$; Phenomenex, Torrance, $\mathrm{CA}$ ). The retained peptides were eluted using Buffer $\mathrm{A}\left(10 \mathrm{mM} \mathrm{KH}_{2} \mathrm{PO}_{4}\right.$ in an aqueous solution of $25 \%$ acetonitrile and acidified to a pH of 3.0 with $\mathrm{H}_{3} \mathrm{PO}_{4}$ ) and Buffer $\mathrm{B}$, where Buffer $B$ was composed of Buffer $A$ with $2 \mathrm{M} \mathrm{KCl}$. The fractions were collected in 1.5-ml microfuge tubes with a flow rate of $1 \mathrm{ml} / \mathrm{min}$. The following chromatographic gradient was applied: 0 25 min 100\% Buffer A; 0 10 min 5\% Buffer B; 10 40 min 5-30\% Buffer B; 40 45 min 30-60\% Buffer B, 45-55 min 60-80\% Buffer B; 55-65 min decreasing to 5\% Buffer B. Fraction collection was started 26 min after the injection with a sample collected every $1 \mathrm{~min}$ to obtain a total of 38 fractions. Eluted fractions were dried in a vacuum concentrator, and each fraction was dissolved in $0.1 \%$ formic acid solution prior to reversedphase nano-LC-tandem mass spectrometry (LC-MS/MS).

\section{LC-MS/MS analysis}

The peptides were dissolved with $50 \mu \mathrm{l}$ of mobile phase $\mathrm{A}\left(\mathrm{H}_{2} \mathrm{O}, 0.1 \%\right.$ formic acid) and loaded onto an Acclaim PePmap C18 reversed-phase column $(75 \mu \mathrm{m} \times 2 \mathrm{~cm}, 3 \mu \mathrm{m}, 100 \AA$, Thermo Scientific) and separated with a reversed-phase $\mathrm{C} 18$ column $(75 \mu \mathrm{m} \times 10 \mathrm{~cm}, 5 \mu \mathrm{m}, 300 \AA$, Agela Technologies $)$ mounted on a Dionex Ultimate 3000 nano-LC system. Peptides were eluted using the following gradient: 0 6 min $5 \%$ Buffer B; 6 6.5 min 10\% Buffer B; 6.5 45 min 10-24\% Buffer B; 45 51 min 24-40\% Buffer B, 51-54 min $40-80 \%$ Buffer $B ; 54-59$ min $80 \%$ Buffer $B ; 59-59.9$ min decreasing to $5 \%$ Buffer $B ; 59.9-65$ min $5 \%$

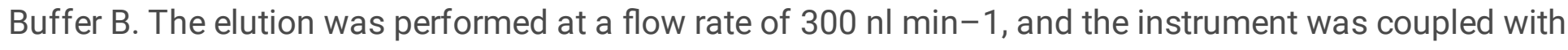
a Q Exactive mass spectrometer (Thermo Fisher Scientific, MA, USA). The eluates directly entered the QExactive MS instrument (Thermo Fisher Scientific, Waltham, MA, USA), and the experiment was performed in positive-ion mode and in a data-dependent manner with full MS scan from 350-2000 m/z, full scan resolution at 70,000, MS/MS scan resolution at 17,500. MS/MS scan with minimum signal threshold $1 \mathrm{E}+5$, and isolation width at $2 \mathrm{Da}$. To evaluate the performance of this mass spectrometry experiment with the iTRAQ-labeled samples, two MS/MS acquisition modes and higher-energy collisional dissociation (HCD) were employed. To optimize the MS/MS acquisition efficiency of HCD, normalized collision energy (NCE) was systemically examined from $20 \%$ and determined to be optimal at $28 \%$ NCE. 


\section{Protein identification and quantification}

Peptide identification and quantification was carried out on PD囚Proteome Discoverer》software, version 1.3 (Thermo), and with the integrated false discovery rate (FDR) analysis function. The data were searched against a protein sequence database downloaded from the BUSCO https://busco.ezlab.org/database. The MS/MS spectra obtained were searched using the following userdefined search parameters: sample type: iTRAQ 8-plex (peptide labeled); cysteine alkylation; digestion: trypsin; instrument: Q-Exactive; species: Brassica carinata; search effort: thorough. For the FDR analysis, the MS/MS spectra were searched against a decoy database to estimate the false discovery rate (FDR) for peptide identification. The decoy database consisted of reversed protein sequences from the Candida albicans database. The resulting data set was auto-bias-corrected to remove any variations caused by unequal mixing during the combination of different labeled samples. Different modification states of the same peptide sequences are considered distinct by the software.

\section{Results}

\section{Phenotypic characterization of BC-P01 and BC-G02}

The total anthocyanin content (TAC) of the two double-haploid (DH) lines BC-P01 (purple leaves) and BCG02 (green leaves) of $B$. carinata were determined by the $\mathrm{pH}$-based color difference method and simplified method (Fig. 1). The leaves of the BC-P01 line were purple from emergence to senescence. The TAC of BC-P01 was $22.7 \mathrm{~g} \cdot 10 \mathrm{~mL} 0.1 \mathrm{~mol} / \mathrm{L}$ hydrochloric acid, which was significantly higher than that that of BC-G02 (0.97 g.10 mL $0.1 \mathrm{~mol} / \mathrm{L}$ hydrochloric acid), as determined by the simplified method $(\mathrm{p}=$ 0.000) (Fig. 1). In addition, the TAC of BC-P01 22.7 was $3.70 \mathrm{mg} \cdot \mathrm{g}^{-1}$, which was significantly higher than that of BC-G02 $\left(0.05 \mathrm{mg} \cdot \mathrm{g}^{-1}\right)$, as determined by the simplified method $(p=0.000)($ Fig. 1).

\section{Protein identification and quantification}

In this study, a total of 35,296 matched spectra were identified from mature leaves of $B$. carinata with different colors using the iTRAQ technique (Supplementary Fig. 1A). After eliminating low-scoring spectra, the reset spectra were matched to 1,7307 peptides; a total of 4,631 proteins were identified from Ethiopian mustard, and 3,442 proteins were quantifiable (Supplementary Fig. $1 \mathrm{~A}$ ). In addition, the relative molecular mass of all the identified proteins was more than $10 \mathrm{kDa}$ (Supplementary Fig. 1B). Most of the identified peptides were unique peptides (Supplementary Fig. 1C). The number of amino acids in the peptide sequences was mainly between 7 and 35 (Supplementary Fig. 1D). The sequence coverage of most of the proteins in Ethiopian mustard leaves was higher than 5\% (Supplementary Fig. 1E). These results indicated that the proteomics analysis is reliable. In addition, we detected 1,837 transcription factors (TFs), including $160 \mathrm{bHLH}$-type proteins, 136 MYBrelated proteins, 125 FAR1-type proteins, 119 B3-type proteins, and 118 NAC-type proteins (Supplementary Fig. 1F). 
Through comparisons between purple leaves and green leaves of Ethiopian mustard, a total of 285 differentially expressed proteins (DEPs) were identified from three biological replications with $p$ value $<0.05$ (Supplementary Table 1). Of the 285 DEPs, 175 are upregulated and 110 are downregulated in the leaves of BC-P01 compared with the leaves of BC-G02. These DEPs between BC-P01 and BC-G01 were revealed by a hierarchical clustering analysis (Supplementary Fig. 2 and Supplementary Table 2).

\section{Functional classification analysis}

Gene Ontology (GO) functional annotation analysis was used to further clarify the functional distributions of the $285 \mathrm{DEPs}$. The results covered a wide range of biological processes, cellular components and molecular functions, which could be classified into 17, 12, and 11 categories, respectively (Fig. 2 and Supplementary Table 3). Some DEPs were assigned to more than one subcategory. In the "biological process" category, the first three classifications included "cellular process" (121 upregulated DEPs (67.98\%) and 62 downregulated DEPs (55.86\%), "metabolic process" (106 upregulated DEPs (59.55\%) and 61 downregulated DEPs (54.95\%) and "response to stimulus" (74 upregulated DEPs (41.57\%) and 41 downregulated DEPs (36.94\%) (Fig. 2 and Supplementary Table 3). In the "cellular component" category, the first three classifications included "cell part" (151 upregulated DEPs (84.83\%) and 83 downregulated DEPs (74.77\%), "organelle" (107 upregulated DEPs (60.11\%) and 63 downregulated DEPs (56.76\%) and "organelle part" (63 upregulated DEPs (35.39\%) and 43 downregulated DEPs (38.74\%) (Fig. 2 and Supplementary Table 2). In the "molecular function" category, the first three classifications included "catalytic activity" (103 upregulated DEPs (57.87\%) and 68 downregulated DEPs (61.26\%), "binding" (107 upregulated DEPs (60.11\%) and 53 downregulated DEPs (47.75\%), and "molecular function regulator" (8 upregulated DEPs (4.49\%) and 5 downregulated DEPs (4.5\%) (Fig. 2 and Supplementary Table 3 ). These results provided primary proteomic information for DEPs between lines BC-P01 and BC-G02 of B. carinata. We also found that the expression of DEPs in BCP01 with purple leaves was significantly higher than the number of DEPs in BC-G02 with green leaves.

In addition, COG annotation of the DEPs was also performed (Fig. 3 and Supplementary Table 4). All these DEPs were divided into 25 categories, and some DEPs were assigned to more than one subcategory. The first three classifications were "Translation, ribosomal structure and biogenesis" (259 DEPs), "General function prediction only" (223), "Posttranslational modification, protein turnover, chaperones" (196), "Amino acid transport and metabolism" (165), "Carbohydrate transport and metabolism" (159) and "Energy production and conversion" (158). Overall, the expression levels of DEPs in different categories in BC-P01 with purple leaves was significantly higher than that in BC-G02 with green leaves.

The main biochemical metabolism and metabolic pathways associated with the DEPs were also described via KEGG pathway database analysis. The results showed that all the annotated proteins were mapped onto 84 KEGG pathways (Supplementary Table 5). The pathways were mainly related to carbon metabolism (including 7 upregulated proteins and 10 downregulated proteins), biosynthesis of amino 
acids (8 upregulated and 2 downregulated proteins), 2-oxocarboxylic acid metabolism (8 upregulated and 0 downregulated proteins), phenylpropanoid biosynthesis (8 upregulated and 0 downregulated proteins), pyruvate metabolism ( 1 upregulated and 6 downregulated proteins), glutathione metabolism ( 6 upregulated and 1 downregulated proteins), plant-pathogen interaction ( 6 upregulated and 1 downregulated proteins), protein processing in endoplasmic reticulum (7 upregulated and 0 downregulated proteins), and so on. Bioinformatics analysis indicated that five metabolic processes, namely, anthocyanin biosynthesis (map00942), carbon metabolism (map01200), 2-oxocarboxylic acid metabolism(map01210), ascorbate and aldarate metabolism (map00053) and tryptophan metabolism (map00380), were the most significantly elevated metabolic processes. Overall, KEGG pathways were identified for 147 upregulated DEPs and 104 downregulated DEPs.

\section{The anthocyanin biosynthesis pathway}

The anthocyanin content was synthesized by one complex metabolic pathway. In this study, three upregulated proteins were identified to be involved in one significant metabolic pathway (map00942, Fig. 4 and Table 1). The fold change values for the proteins TRINITY_DN5961_c0_g2, TRINITY_DN5961_c0_g1 and TRINITY_DN8427_c0_g1 were 2.13, 2.07 and 2.32, respectively. These proteins correspond to two anthocyanin 5-0-glucosyltransferases and an anthocyanin 2"-0xylosyltransferase, and the genes that encode these proteins are UGT75C1 and UGT79B1, respectively.

\section{Discussion}

The synthesized plant TACs, including mainly cyanidin, delphinidin, petunidin, peonidin, and pelargonidin, can determine plant organ color at specific $\mathrm{pH}$ values, which is why the Ethiopian mustard leaves in this study were purple in color. Three upregulated proteins corresponding to the two genes UGT75C1 and UGT79B1 were identified by iTRAQ. The protein expression levels in BC-P01 with purple leaves was more than two times that in BC-G02 with green leaves. These two genes should be the key genes associated with the color difference between lines BC-P01 and BC-G02 of B. carinata. UGT75C1 and UGT79B1 are involved in the complex metabolic pathways of TAC metabolic synthesis. UGT79B1 (At5g54060), encoding anthocyanin 3-0-glucoside: 2"-0-xylosyltransferase, together with At3AT1 and At3AT2 (At1g03490 and At1g03495), is responsible for the glycosylation and acylation of cyanidin 3-0-glucoside (Cy3G) to produce cyanidin 3-0-[2"-0-(xylosyl)-6"-0-(p-coumaroyl) glucoside] [44-46]. Then, the cyanidin 3-0-[2"-0-(xylosyl)-6"-0-(p-coumaroyl) glucoside] can be glucosylated by UGT75C1 (At4g14090), encoding anthocyanin 5-0-glucosyltransferase, to produce cyanidin 3-0-[2"-0-(xylosyl)-6"-0-(pcoumaroyl) glucoside] 5-0-glucoside [46, 47]. These studies indicate that UGT75C1 and UGT79B1 play an important role in TAC synthesis. By utilization of high-throughput RNA sequencing, the expression of UGT75C1 and UGT79B1 was found to be positively correlated with TAC biosynthesis $[47,48]$. In addition, the expression of UGT75C1 and UGT79B1 was found to be significantly high in purple buds and leaves of tea [49] and in light purple petals compared with white petals [50], respectively. These results are consistent with this study because the protein expression level of UGT75C1 and UGT79B1 was 
upregulated in BC-P01 compared with BC-G02. To date, almost no studies related to UGT75C1 and UGT79B 1 expression at the protein level have been reported. The proteins identified in this study by ITRAQ can provide useful information for studying the metabolic pathway of TAC synthesis in B. carinata and other species in Brassiceae.

The TAC metabolic pathway can be regulated by primary metabolism, including sucrose, starch, and hexose sugars. Sucrose is generally the major end product of photosynthetic carbon metabolism, and in most plants, it is the predominant form of carbon transported to the heterotrophic tissues [51, 52]. The significant metabolic pathway of the carbon metabolism pathway may be the important reason for the color difference between BC-P01 and BC-G02 in this study. Sucrose could provide precursors and sugar moieties for TAC synthesis. In addition, sucrose can specifically induce the TAC biosynthetic pathway for signaling, and these signal transduction pathways may lead to the activation or inactivation of gene expression [20,53-55]. The degradation of starch can contribute to anthocyanin accumulation in the tuberous root of purple sweet potato [56]. The TAC metabolic pathway can be affected by some other hormones, such as methyl jasmonate [55], GA [57], abscisic acid [58, 59], BPA [60], ethylene [61], and hematin [62]. TAC synthesis can also be regulated by fertilizers [63], such as nitrogen $[64,65]$ and phosphate [66]. Light [67-70], short-day signals [71] and low temperature [72] can also affect the TAC biosynthesis pathway. Therefore, all of the pathways involved in the synthesis, degradation and absorption of these factors can regulate TAC biosynthesis in this study.

The genetic transcription of genes in the TAC biosynthetic pathway can be regulated by a combination of transcription factors (TFs). The regulatory effects of TFs on TAC biosynthesis have been reported in many studies. The TFs include MYB, bHLH, and WDR. In Arabidopsis thaliana, the PAP1-GL3-TTG1 complex can regulate the TFs of MYB and bHLH [73]. In addition, the reduction of MYB expression can reduce the expression levels of DFR, LDOX, GST12, F3'H and UGT75C1 in the TAC pathway [74]. UGT75C1 is also upregulated by $P A P 1$, encoding an MYB transcription factor [47]. As a whole, the TF complex (TT2, TT8, and TTG1) and SmMYB1, an R2R3 MYB transcription factor, can regulate all anthocyanin pathway genes $[73,75]$. In this study, we identified thousands of TFs, including TFs for TAC synthesis, which might be involved in the regulation of genes in the TAC metabolic pathway.

\section{Conclusions}

In this study, an iTRAQ-based proteomic study was carried out to investigate the protein expression profile of the purple-leaved line of $B$. carinata in comparison with that of the green-leaved line. Identified DEPs by bioinformatics analysis were mainly associated with "translation, ribosomal structure and biogenesis", "general function prediction only", "posttranslational modification, protein turnover, chaperones", "amino acid transport and metabolism", "carbohydrate transport and metabolism" and "energy production and conversion". For anthocyanin biosynthesis, three DEPs, corresponding to two genes UGT75C1 and UGT79B1, might play an important role. Our results provide insights into whole-proteomic profiles and regulatory genes that may be responsible for anthocyanin biosynthesis in $B$. carinata, and suggest that 
future efforts will be directed towards understanding how these candidate genes regulate the anthocyanin biosynthesis pathways in this species.

\section{Abbreviations}

iTRAQ: isobaric tags for relative and absolute quantification; TCA: trichloroacetic acid; IAM: iodoacetamide; HCD: higher-energy collisional dissociation; NCE: normalized collision energy; PD: proteome discoverer; FDR: false discovery rate; TAC: total anthocyanin content; DEPs: differentially expressed proteins; GO: gene ontology; COG: clusters of orthologous group; KEGG: kyoto encyclopedia of genes and genomes; TFs: transcription factors

\section{Declarations}

\section{Acknowledgments}

Not applicable.

\section{Ethics approval consent to participate}

Not applicable.

\section{Authors' contributions}

ET conceived and designed the experiments. TW, SG, WW, YB, RY and ANK performed the experiments. TW, SG and ET analyzed the data and wrote the paper. ET, SG, KY, JZ, YJ and JM revised the paper. All authors read and approved the final manuscript.

\section{Funding}

The financial of this research was supported by the National Natural Science Foundation of China (grant no. 31560422), Agricultural Science and Technology Support Program of Guizhou Province (Qiankehe Zhicheng no. [2019]2396), Science and Technology Foundation of Guizhou Province of China (grant no. Qiankehe J zi [2015]2052), Scientific Research Foundation for Returned Scholars, Ministry of Education of China (grant no. Jiaowaisiliu [2015]1098), Foundation of Guizhou University (grant no. Guidarenjihezi [2014]14), and Construction Program of Biology First-class Discipline in Guizhou (grant no. GNYL[2017]009).

\section{Availability of data and materials}


All data sets generated as part of this study are available at the additional files of this study.

\section{Consent for publication}

Not applicable.

\section{Competing interests}

The authors declare that they have no competing interests.

\section{Author details}

${ }^{1}$ Agricultural College of Guizhou University, Guizhou University, Guiyang 550025, China. ${ }^{2}$ Institute of Rapeseed Research, Guizhou Academy of Agricultural Science, Guiyang 550018, China. ${ }^{3}$ National Key Laboratory of Crop Genetic Improvement, Huazhong Agricultural University, Wuhan 430070, China. ${ }^{4}$ Anhui Academy of Agricultural Science, Hefei 230001, China.

\section{References}

1.Warwick SI: Brassicaceae in Agriculture. In: Genetics and Genomics of the Brassicaceae. Edited by Schmidt R, Bancroft I. New York, NY: Springer New York; 2011: 33-65.

2.Guo S, Zou J, Li R, Long Y, Chen S, Meng J: A genetic linkage map of Brassica carinata constructed with a doubled haploid population. Theoretical and Applied Genetics 2012, 125(6):1113-1124.

3.Warwick SI, Gugel RK, McDonald T, Falk KC: Genetic Variation of Ethiopian Mustard (Brassica carinata A. Braun) Germplasm in Western Canada. Genetic Resources and Crop Evolution 2006, 53(2):297-312.

4.Hu J, Quiros CF: APPLICATION OF MOLECULAR MARKERS AND CYTOGENETIC STOCKS TO BRASSICA GENETICS, BREEDING AND EVOLUTION. Acta Horticulturae 1996(407):79-86.

5.U: Genome analysis in Brassica with special reference to the experimental formation of B. napus and peculiar mode of fertilization. Journal of Japanese Bontany 1935, 7.

6.LUKENS LN, QUIJADA PA, UDALL J, PIRES JC, SCHRANZ ME, OSBORN TC: Genome redundancy and plasticity within ancient and recent Brassica crop species. Biological Journal of the Linnean Society 2004, 82(4):665-674.

7.Song K, Osborn TC, Williams $\mathrm{PH}$ : Brassica taxonomy based on nuclear restriction fragment length polymorphisms (RFLPS). Theoretical and Applied Genetics 1990, 79(4):497-506. 
8.Alemayehu N, Becker $\mathrm{H}$ : Genotypic diversity and patterns of variation in a germplasm material of Ethiopian mustard (Brassica carinata A. Braun). Genetic Resources and Crop Evolution 2002, 49(6):573582.

9.Abebe D, Dawit T, Getahun M, Debritu B: Ethiopia's genetic resources of oilseeds. In: Proc. 1 National Oilseeds Workshop. Institute of Agricultural Research, Addis Ababa, Ethiopia, pp 12-171992.

10.Jiang Y, Tian E, Li R, Chen L, Meng J: Genetic diversity of Brassica carinata with emphasis on the interspecific crossability with B. rapa. Plant Breeding 2007, 126(5):487-491.

11.Taylor DC, Falk KC, Palmer CD, Hammerlindl J, Babic V, Mietkiewska E, Jadhav A, Marillia E-F, Francis T, Hoffman T et al: Brassica carinata-a new molecular farming platform for delivering bio-industrial oil feedstocks: case studies of genetic modifications to improve very long-chain fatty acid and oil content in seeds. Biofuels, Bioproducts and Biorefining 2010, 4(5):538-561.

12.Getinet A, Rakow G, Downey RK: Agronomic performance and seed quality of Ethiopian mustard in Saskatchewan. Canadian Journal of Plant Science 1996, 76(3):387-392.

13.Meng J, Shi S, Gan L, Li Z, Qu X: The production of yellow-seeded Brassica napus (AACC) through crossing interspecific hybrids of $B$. campestris $(A A)$ and B. carinata $(B B C C)$ with $B$. napus. Euphytica 1998, 103(3):329-333.

14.Tian E, Jiang Y, Chen L, Zou J, Liu F, Meng J: Synthesis of a Brassica trigenomic allohexaploid (B. carinata $\times$ B. rapa) de novo and its stability in subsequent generations. Theoretical and Applied Genetics 2010, 121(8):1431-1440.

15.Xiao Y, Chen L, Zou J, Tian E, Xia W, Meng J: Development of a population for substantial new type Brassica napus diversified at both A/C genomes. Theoretical and Applied Genetics 2010, 121(6):11411150.

16.Zou J, Zhu J, Huang S, Tian E, Xiao Y, Fu D, Tu J, Fu T, Meng J: Broadening the avenue of intersubgenomic heterosis in oilseed Brassica. Theoretical and Applied Genetics 2010, 120(2):283-290.

17.Zou J, Hu D, Mason AS, Shen X, Wang X, Wang N, Grandke F, Wang M, Chang S, Snowdon RJ et al: Genetic changes in a novel breeding population of Brassica napus synthesized from hundreds of crosses between B. rapa and B. carinata. Plant biotechnology journal 2018, 16(2):507-519.

18.Zou J, Raman H, Guo S, Hu D, Wei Z, Luo Z, Long Y, Shi W, Fu Z, Du D et al: Constructing a dense genetic linkage map and mapping QTL for the traits of flower development in Brassica carinata. Theoretical and Applied Genetics 2014, 127(7):1593-1605.

19.Zhang W, Hu D, Raman R, Guo S, Wei Z, Shen X, Meng J, Raman H, Zou J: Investigation of the Genetic Diversity and Quantitative Trait Loci Accounting for Important Agronomic and Seed Quality Traits in Brassica carinata. In: Frontiers in plant science. vol. 8; 2017: 615. 
20.Li D, Zhang X, Xu Y, Li L, Aghdam MS, Luo Z: Effect of exogenous sucrose on anthocyanin synthesis in postharvest strawberry fruit. Food chemistry 2019, 289:112-120.

21.Pojer E, Mattivi F, Johnson D, Stockley CS: The Case for Anthocyanin Consumption to Promote Human Health: A Review. Comprehensive Reviews in Food Science and Food Safety 2013, 12(5):483-508.

22.Prior RL, Xianli W: Anthocyanins: structural characteristics that result in unique metabolic patterns and biological activities. Free Radic Res 2006, 40(10):1014-1028.

23.Mazza G, Miniati E: Anthocyanins in fruits, vegetables, and grains. Anthocyanins in Fruits Vegetables \& Grains 1993.

24.Turturica M, Oancea A-M, Rapeanu G, Bahrim G: Anthocyanins: Naturally occuring fruit pigments with functional properties. Annals of the University Dunarea de Jos of Galati Fascicle VI: Food Technology 2015, 39:9-24.

25.Khoo HE, Azlan A, Tang ST, Lim SM: Anthocyanidins and anthocyanins: colored pigments as food, pharmaceutical ingredients, and the potential health benefits. Food \& Nutrition Research 2017, 61(1):1361779.

26.Dong T, Han R, Yu J, Zhu M, Zhang Y, Gong Y, Li Z: Anthocyanins accumulation and molecular analysis of correlated genes by metabolome and transcriptome in green and purple asparaguses (Asparagus officinalis, L.). Food chemistry 2019, 271:18-28.

27.Gamel TH, Wright AJ, Tucker AJ, Pickard M, Rabalski I, Podgorski M, Di llio N, O'Brien C, Abdel-Aal ESM: Absorption and metabolites of anthocyanins and phenolic acids after consumption of purple wheat crackers and bars by healthy adults. Journal of Cereal Science 2019, 86:60-68.

28.Sun D, Huang S, Cai S, Cao J, Han P: Digestion property and synergistic effect on biological activity of purple rice (Oryza sativa L.) anthocyanins subjected to a simulated gastrointestinal digestion in vitro. Food research international 2015, 78:114-123.

29.Ferarsa S, Zhang W, Moulai-Mostefa N, Ding L, Jaffrin MY, Grimi N: Recovery of anthocyanins and other phenolic compounds from purple eggplant peels and pulps using ultrasonic-assisted extraction. Food and Bioproducts Processing 2018, 109:19-28.

30.Lao F, Giusti MM: Quantification of Purple Corn (Zea mays L.) Anthocyanins Using Spectrophotometric and HPLC Approaches: Method Comparison and Correlation. Food Analytical Methods 2016, 9(5):13671380.

31.Zhang H, Hassan YI, Renaud J, Liu R, Yang C, Sun Y, Tsao R: Bioaccessibility, bioavailability, and antiinflammatory effects of anthocyanins from purple root vegetables using mono- and co-culture cell models. Molecular nutrition \& food research 2017, 61(10):1600928. 
32.Szymanowska U, Złotek U, Karaś M, Baraniak B: Anti-inflammatory and antioxidative activity of anthocyanins from purple basil leaves induced by selected abiotic elicitors. Food chemistry 2015, 172:71-77.

33.Lai Y-S, Li S, Tang Q, Li H-X, Chen S-X, Li P-W, Xu J-Y, Xu Y, Guo X: The Dark-Purple Tea Cultivar 'Ziyan' Accumulates a Large Amount of Delphinidin-Related Anthocyanins. Journal of agricultural and food chemistry 2016, 64(13):2719-2726.

34.Ou L-j, Zhang Z-q, Dai X-z, Zou X-x: Photooxidation Tolerance Characters of a New Purple Pepper. PloS one 2013, 8(5):e63593.

35.Yan C, An G, Zhu T, Zhang W, Zhang L, Peng L, Chen J, Kuang H: Independent activation of the BoMYB2 gene leading to purple traits in Brassica oleracea. Theoretical and Applied Genetics 2019, 132(4):895-906.

36.Li H, Zhu L, Yuan G, Heng S, Yi B, Ma C, Shen J, Tu J, Fu T, Wen J: Fine mapping and candidate gene analysis of an anthocyanin-rich gene, BnaA.PL 1, conferring purple leaves in Brassica napus L. Molecular Genetics and Genomics 2016, 291(4):1523-1534.

37.Wang C, Li H, Li Y, Meng Q, Xie F, Xu Y, Wan Z: Genetic characterization and fine mapping BrCER4 in involved cuticular wax formation in purple cai-tai (Brassica rapa L. var. purpurea). Molecular Breeding 2019, 39(1):12.

38.Zhao Z, Xiao L, Xu L, Xing X, Tang G, Du D: Fine mapping the BjPl1 gene for purple leaf color in B2 of Brassica juncea L. through comparative mapping and whole-genome re-sequencing. Euphytica 2017, 213(4):80.

39. Wiese S, Reidegeld KA, Meyer HE, Warscheid B: Protein labeling by iTRAQ: A new tool for quantitative mass spectrometry in proteome research. PROTEOMICS 2007, 7(3):340-350.

40.Wang W, Zhang D, Yu S, Liu J, Wang D, Zhang F, Yu Y, Zhao X, Lu G, Su T: Mapping the BrPur gene for purple leaf color on linkage group A03 of Brassica rapa. Euphytica 2014, 199(3):293-302.

41.Cheng GW, Breen PJ: Activity of Phenylalanine Ammonia-Lyase (PAL) and Concentrations of Anthocyanins and Phenolics in Developing Strawberry Fruit. Journal of the American Society for Horticultural Science 1991, 116(5).

42.Wang J, Zhang H, Zhang J, Zhang H, Liu B, Hou Y, Qiu N: Changes of Anthocyanin Content and Photosynthetic Function of Purple Leaf Plum Leaves during Spring Maturation. FORESTRY SCIENCE \& TECHNOLOGY 2018, 43(03):13-17.

43.Yu X: Analysis and Study of Anthocyanins in Plant Leave Modern Instruments 2000(04):37-38. 
44.Yonekura-Sakakibara K, Fukushima A, Nakabayashi R, Hanada K, Matsuda F, Sugawara S, Inoue E, Kuromori T, Ito T, Shinozaki K et al: Two glycosyltransferases involved in anthocyanin modification delineated by transcriptome independent component analysis in Arabidopsis thaliana. The Plant Journal 2012, 69(1):154-167.

45.Luo J, Nishiyama Y, Fuell C, Taguchi G, Elliott K, Hill L, Tanaka Y, Kitayama M, Yamazaki M, Bailey P et al: Convergent evolution in the BAHD family of acyl transferases: identification and characterization of anthocyanin acyl transferases from Arabidopsis thaliana. The Plant Journal 2007, 50(4):678-695.

46.Miyahara T, Sakiyama R, Ozeki Y, Sasaki N: Acyl-glucose-dependent glucosyltransferase catalyzes the final step of anthocyanin formation in Arabidopsis. Journal of Plant Physiology 2013, 170(6):619-624.

47.Tohge T, Nishiyama Y, Hirai MY, Yano M, Nakajima J-i, Awazuhara M, Inoue E, Takahashi H, Goodenowe DB, Kitayama M et al: Functional genomics by integrated analysis of metabolome and transcriptome of Arabidopsis plants over-expressing an MYB transcription factor. The Plant Journal 2005 , 42(2):218-235.

48.Bowles D: A multigene family of glycosyltransferases in a model plant, Arabidopsis thaliana. Biochemical Society Transactions 2002, 30(2):301-306.

49.Guo Y, Chang X, Zhu C, Zhang S, Li X, Fu H, Chen C, Lin Y, Lai Z: De novo transcriptome combined with spectrophotometry and gas chromatography-mass spectrometer (GC-MS) reveals differentially expressed genes during accumulation of secondary metabolites in purple-leaf tea (Camellia sinensis cv Hongyafoshou). The Journal of Horticultural Science and Biotechnology 2019, 94(3):349-367.

50.Chen D, Liu Y, Pan Q, Li F, Zhang Q, Ge X, Li Z: De novo transcriptome assembly, gene expressions and metabolites for flower color variation of two garden species in Brassicaceae. Scientia Horticulturae 2018, 240:592-602.

51.Ziegler H: Nature of Transported Substances. In: Transport in Plants I: Phloem Transport. Edited by Zimmermann MH, Milburn JA. Berlin, Heidelberg: Springer Berlin Heidelberg; 1975: 59-100.

52.Chiou T-J, Bush DR: Sucrose is a signal molecule in assimilate partitioning. Proceedings of the National Academy of Sciences 1998, 95(8):4784-4788.

53.Solfanelli C, Poggi A, Loreti E, Alpi A, Perata P: Sucrose-Specific Induction of the Anthocyanin Biosynthetic Pathway in Arabidopsis. Plant physiology 2006, 140(2):637-646.

54.Ai TN, Naing AH, Arun M, Lim S-H, Kim CK: Sucrose-induced anthocyanin accumulation in vegetative tissue of Petunia plants requires anthocyanin regulatory transcription factors. Plant Science 2016, 252:144-150.

55.Kim SH, Kim Y-S, Jo YD, Kang S-Y, Ahn J-W, Kang B-C, Kim J-B: Sucrose and methyl jasmonate modulate the expression of anthocyanin biosynthesis genes and increase the frequency of flower-color 
mutants in chrysanthemum. Scientia Horticulturae 2019, 256:108602.

56.Wang S, Pan D, Lv X, Song X, Qiu Z, Huang C, Huang R, Chen W: Proteomic approach reveals that starch degradation contributes to anthocyanin accumulation in tuberous root of purple sweet potato. Journal of proteomics 2016, 143:298-305.

57.Tan H, Man C, Xie Y, Yan J, Chu J, Huang J: A Crucial Role of GA-Regulated Flavonol Biosynthesis in Root Growth of Arabidopsis. Molecular plant 2019, 12(4):521-537.

58.Karppinen K, Tegelberg P, Häggman H, Jaakola L: Abscisic Acid Regulates Anthocyanin Biosynthesis and Gene Expression Associated With Cell Wall Modification in Ripening Bilberry (Vaccinium myrtillus L.) Fruits. Frontiers in plant science 2018, 9(1259).

59.Sun Y, Liu Q, Xi B, Dai H: Study on the regulation of anthocyanin biosynthesis by exogenous abscisic acid in grapevine. Scientia Horticulturae 2019, 250.294-301.

60.Kim D, Bahmani R, Ko J-H, Hwang S: Development of bisphenol A (BPA)-sensing indicator Arabidopsis thaliana which synthesizes anthocyanin in response to BPA in leaves. Ecotoxicology and Environmental Safety 2019, 170:627-634.

61.Kumar D, Sarmah BK, Das PK: Ethylene mediates repression of anthocyanin accumulation in black rice pericarps in the absence of light. Journal of Plant Physiology 2019, 236:34-38.

62.Li Y, Li H, Wang F, Li J, Zhang Y, Wang L, Gao J: Comparative Transcriptome Analysis Reveals Effects of Exogenous Hematin on Anthocyanin Biosynthesis during Strawberry Fruit Ripening. International Journal of Genomics 2016, 2016:14.

63.Jezek M, Zörb C, Merkt N, Geilfus CM: Anthocyanin management in fruits by fertilization. Journal of Agricultural \& Food Chemistry 2018, 66(4):acs.jafc.7b03813.

64.Luo J, Wang X, Feng L, Li Y, He J-X: The mitogen-activated protein kinase kinase 9 (MKK9) modulates nitrogen acquisition and anthocyanin accumulation under nitrogen-limiting condition in Arabidopsis. Biochemical and biophysical research communications 2017, 487(3):539-544.

65.Truong HA, Lee WJ, Jeong CY, Trịnh CS, Lee S, Kang C-S, Cheong Y-K, Hong S-W, Lee H: Enhanced anthocyanin accumulation confers increased growth performance in plants under low nitrate and high salt stress conditions owing to active modulation of nitrate metabolism. Journal of Plant Physiology 2018, 231:41-48.

66.Wang ZQ, Zhou X, Dong L, Guo J, Chen Y, Zhang Y, Wu L, Xu M: iTRAQ-based analysis of the Arabidopsis proteome reveals insights into the potential mechanisms of anthocyanin accumulation regulation in response to phosphate deficiency. Journal of proteomics 2018, 184:39-53. 
67.Zhang KM, Guo ML, He D, Wu RH, Li YH: The Inhibition Effect and Excessive Carbon Flux Resulting from Blocking Anthocyanin Biosynthesis Under Darkness in Begonia semperflorens. Journal of Plant Growth Regulation 2016, 35(1):22-30.

68.Zhang H-N, Li W-C, Wang H-C, Shi S-Y, Shu B, Liu L-Q, Wei Y-Z, Xie J-H: Transcriptome Profiling of Light-Regulated Anthocyanin Biosynthesis in the Pericarp of Litchi. Frontiers in plant science 2016, 7(963).

69.Li J, Ren L, Gao Z, Jiang M, Liu Y, Zhou L, He Y, Chen H: Combined transcriptomic and proteomic analysis constructs a new model for light-induced anthocyanin biosynthesis in eggplant (Solanum melongena L.). Plant, cell \& environment 2017, 40(12):3069-3087.

70.Xu P, Zawora C, Li Y, Wu J, Liu L, Liu Z, Cai R, Lian H: Transcriptome sequencing reveals role of light in promoting anthocyanin accumulation of strawberry fruit. Plant Growth Regulation 2018, 86(1):121-132.

71.Zhang KM, Wang JW, Guo ML, Du WL, Wu RH, Wang X: Short-day signals are crucial for the induction of anthocyanin biosynthesis in Begonia semperflorens under low temperature condition. Journal of Plant Physiology 2016, 204:1-7.

72.Bhatia C, Pandey A, Gaddam SR, Hoecker U, Trivedi PK: Low Temperature-Enhanced Flavonol Synthesis Requires Light-Associated Regulatory Components in Arabidopsis thaliana. Plant and Cell Physiology 2018, 59(10):2099-2112.

73.Zhu Z, Wang H, Wang Y, Guan S, Wang F, Tang J, Zhang R, Xie L, Lu Y: Characterization of the cis elements in the proximal promoter regions of the anthocyanin pathway genes reveals a common regulatory logic that governs pathway regulation. Journal of experimental botany 2015, 66(13):37753789.

74.Gonzalez A, Zhao M, Leavitt JM, Lloyd AM: Regulation of the anthocyanin biosynthetic pathway by the TTG1/bHLH/Myb transcriptional complex in Arabidopsis seedlings. The Plant Journal 2008, 53(5):814-827.

75.Zhang Y, Chu G, Hu Z, Gao Q, Cui B, Tian S, Wang B, Chen G: Genetically engineered anthocyanin pathway for high health-promoting pigment production in eggplant. Molecular Breeding 2016, 36(5):54.

\section{Table 1}

Table 1 Relative differences in the expression levels of the proteins involved in the anthocyanin biosynthesis pathway between purple-leaved and green-leaved Ethiopian mustard. 


\begin{tabular}{|c|c|c|c|c|c|}
\hline $\begin{array}{c}\text { Serial } \\
\text { number }\end{array}$ & Protein ID & Description & $\begin{array}{l}\text { Gene } \\
\text { name }\end{array}$ & $\begin{array}{c}\text { Fold change } \llbracket \mathrm{BC}-\mathrm{P} 01 \\
\text { versus } \mathrm{BC}-\mathrm{G} 02 \square\end{array}$ & $\begin{array}{c}\mathrm{P}- \\
\text { value }\end{array}$ \\
\hline 1 & TRINITY_DN5961_c0_g2 & \multirow{2}{*}{$\begin{array}{l}\text { anthocyanin 5-O- } \\
\text { glucosyltransferase }\end{array}$} & UGT75C1 & 2.13 & 0.000 \\
\hline 2 & TRINITY_DN5961_c0_g1 & & UGT75C1 & 2.07 & 0.001 \\
\hline 3 & TRINITY_DN8427_c0_g1 & $\begin{array}{l}\text { anthocyanin 2"-O- } \\
\text { xylosyltransferase }\end{array}$ & UGT79B1 & 2.32 & 0.007 \\
\hline
\end{tabular}

\section{Figures}

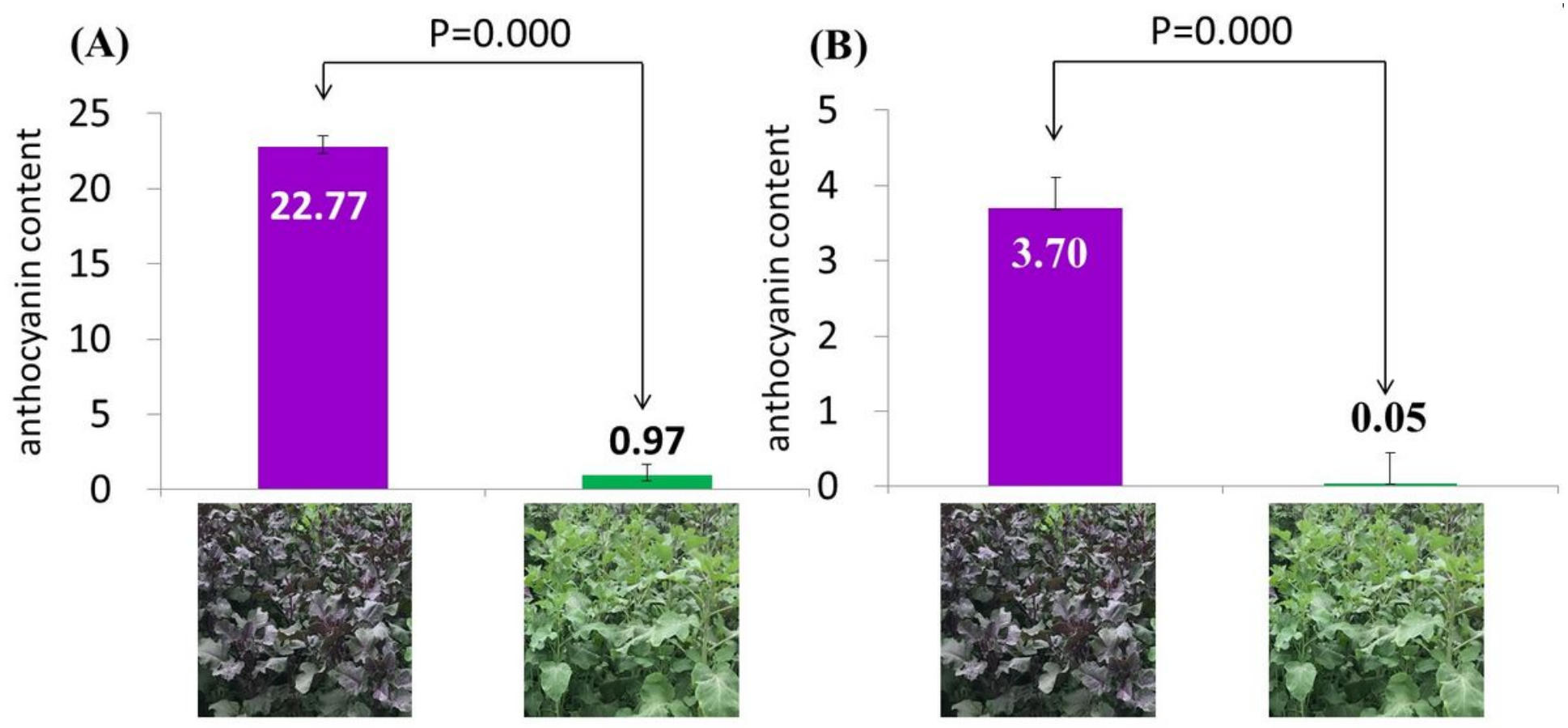

\section{Figure 1}

The total anthocyanin content determined by the simplified method $(A, g \cdot 10 \mathrm{~mL} 0.1 \mathrm{~mol} / \mathrm{L}$ hydrochloric acid) and $\mathrm{pH}$ differential method $(B, \mathrm{mg} \cdot \mathrm{g}-1)$ in Ethiopian mustard (B. carinata) lines with purple and green leaves. 


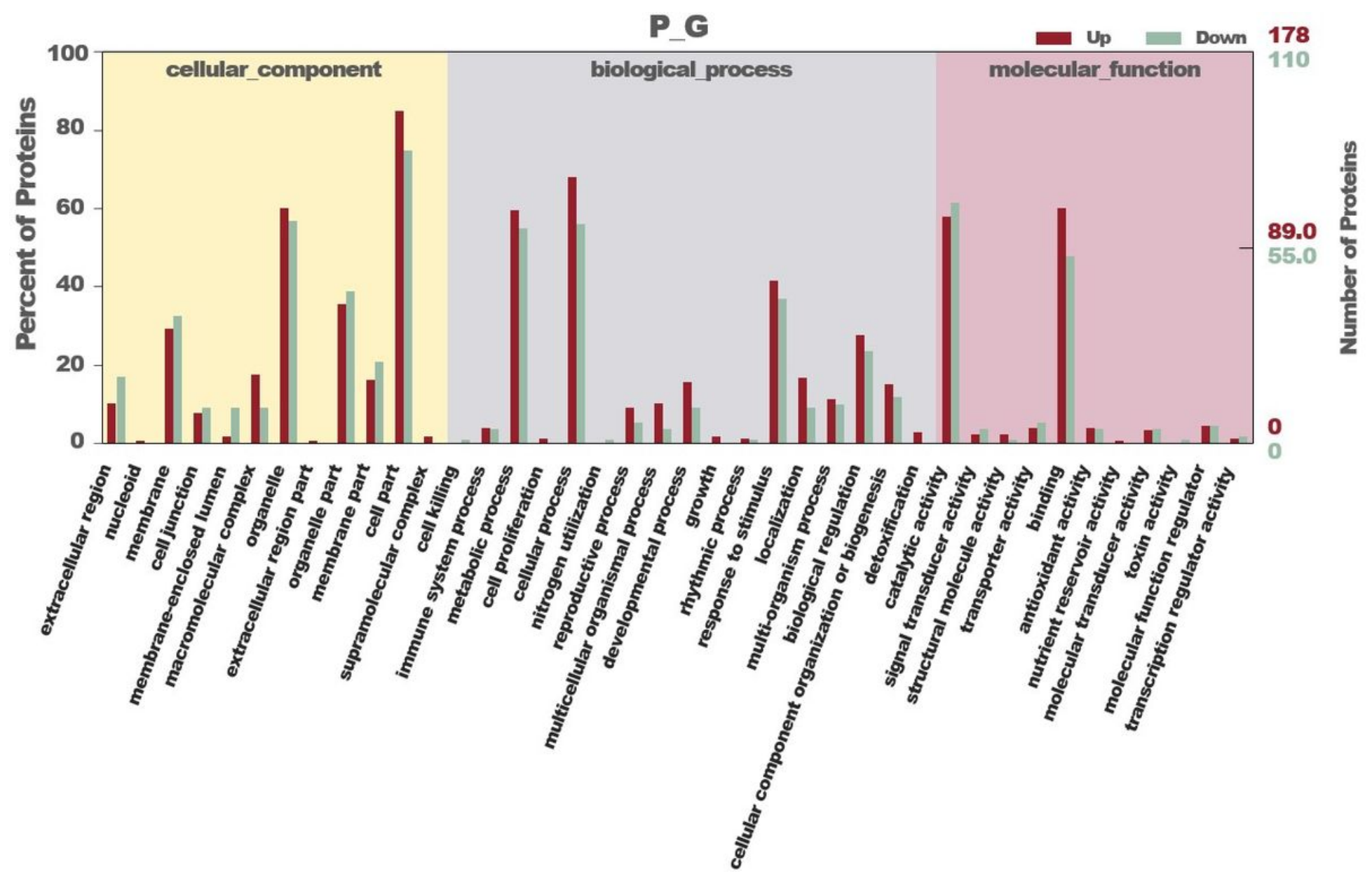

Figure 2

Gene Ontology (GO) functional annotation analysis of the DEPs, covering a wide range of biological processes, cellular components and molecular functions. 


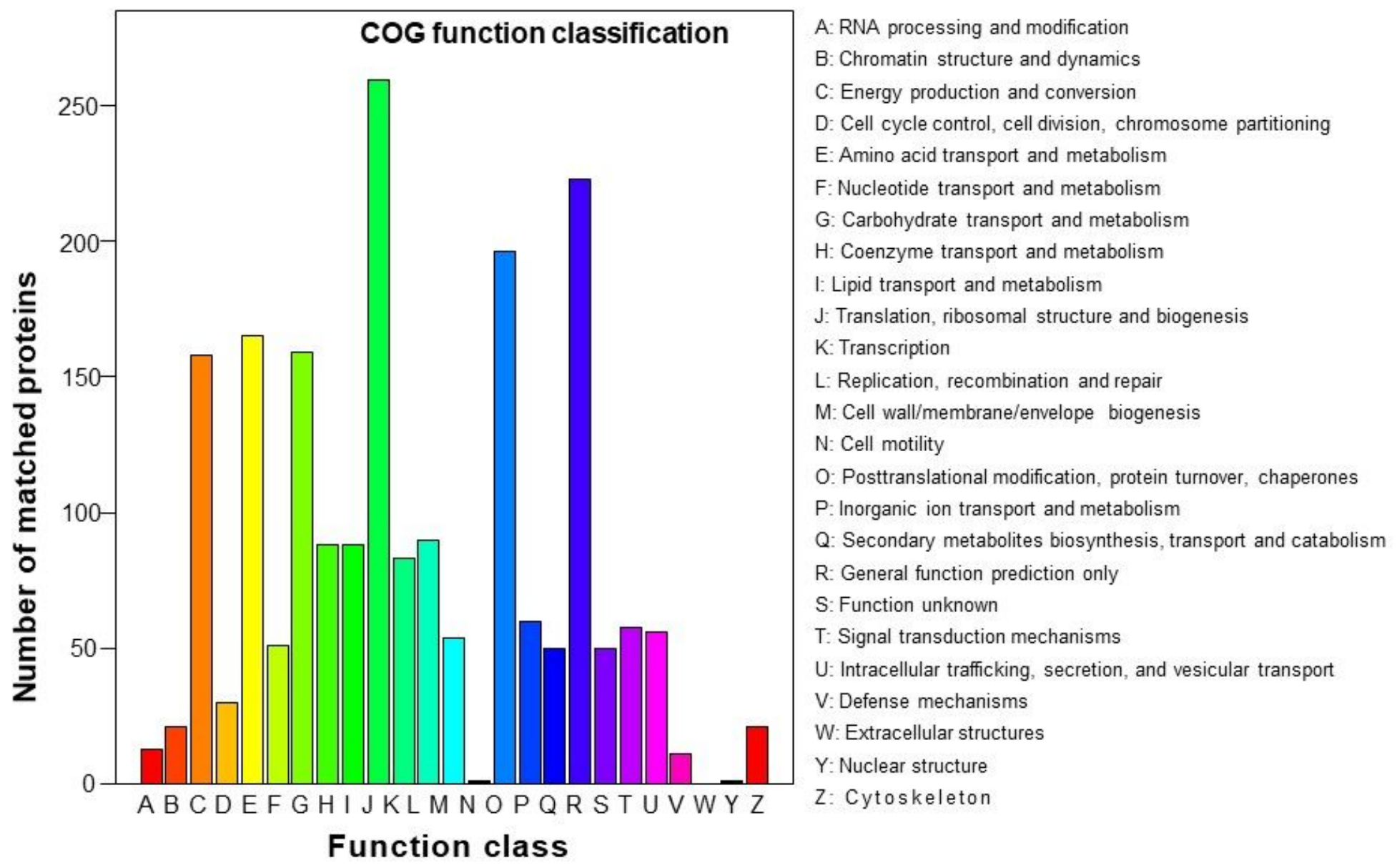

Figure 3

COG annotation of the DEPs, which are divided into 25 categories. 


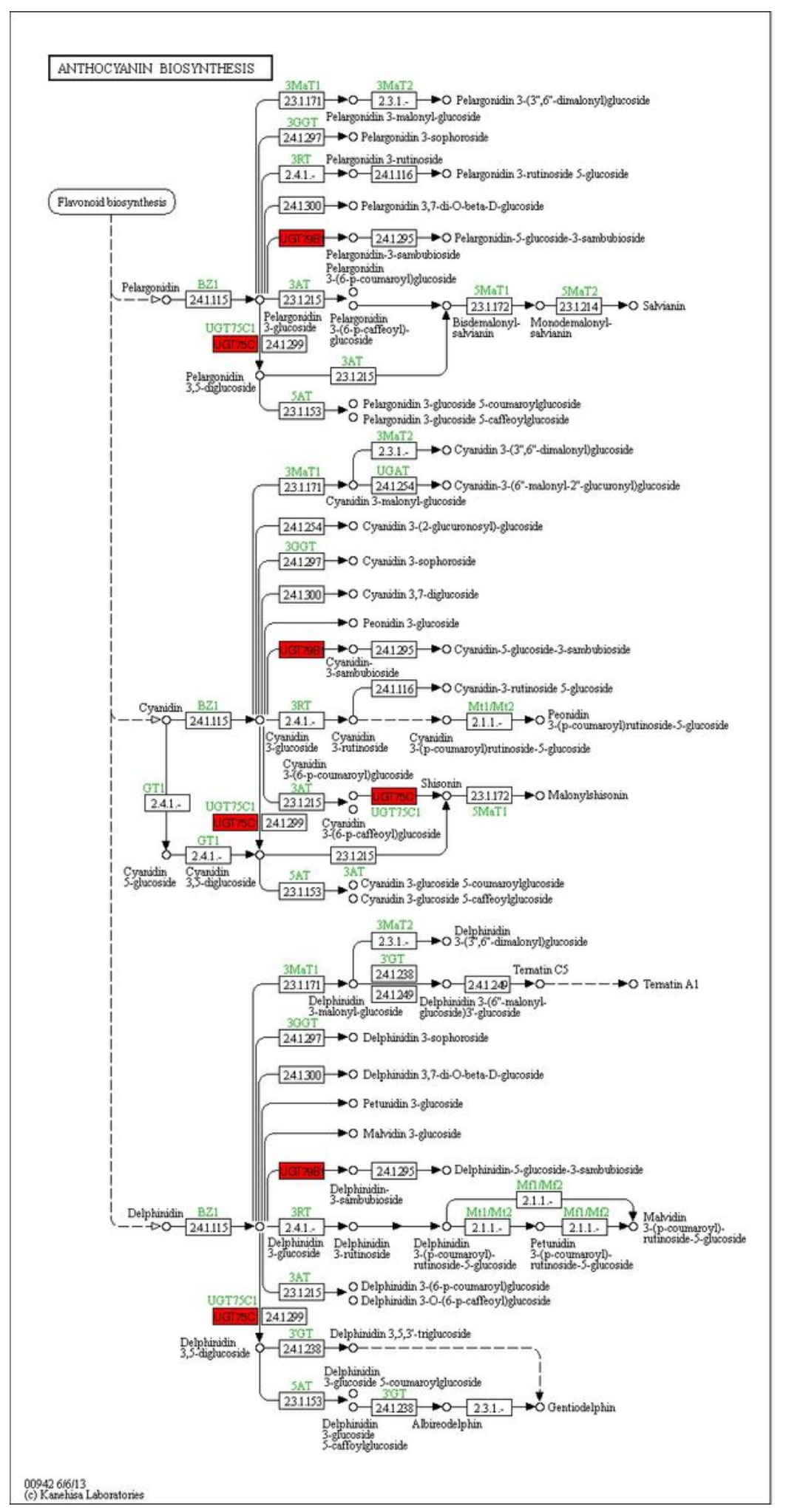

\section{Figure 4}

The anthocyanin metabolic pathway, including three upregulated proteins identified in this study.

\section{Supplementary Files}

This is a list of supplementary files associated with this preprint. Click to download. 
- SupplementaryTable1DEPs.xlsx

- SupplementaryTable5KEGG.xIsx

- SupplementaryFig.1.docx

- SupplementaryTable2TF.xls

- SupplementaryTable4COG.xIsx

- SupplementaryFig.2Hierarchicalclustering.docx

- SupplementaryTable3G0.xIsx 